Kansas State University Libraries

New Prairie Press

\title{
SIMPLE STEP-STRESS TESTING WITH COVARIATE IN AGRICULTURE
}

Larry Cleair

Imad H. Khamis

Mohamad Al-Haj Ebrahem

Follow this and additional works at: https://newprairiepress.org/agstatconference

Part of the Agriculture Commons, and the Applied Statistics Commons

\section{(c) (1) $\Theta$}

This work is licensed under a Creative Commons Attribution-Noncommercial-No Derivative Works 4.0 License.

\section{Recommended Citation}

Cleair, Larry; Khamis, Imad H.; and Ebrahem, Mohamad AI-Haj (2003). "SIMPLE STEP-STRESS TESTING WITH COVARIATE IN AGRICULTURE," Conference on Applied Statistics in Agriculture. https://doi.org/ $10.4148 / 2475-7772.1188$

This is brought to you for free and open access by the Conferences at New Prairie Press. It has been accepted for inclusion in Conference on Applied Statistics in Agriculture by an authorized administrator of New Prairie Press. For more information, please contact cads@k-state.edu. 


\title{
Simple Step-Stress Testing with Covariate in Agriculture
}

\author{
Larry Cleair Southeast Missouri State University \\ Dr. Imad H. Khamis Southeast Missouri State University \\ Mohamad Al-Haj Ebrahem Kansas State University
}

\begin{abstract}
In industry product testing can be an expensive and time-consuming process. Testing design changes in long-lived products could cause lengthy delays in product introduction or improvement. As an alternative, accelerated life testing can quickly yield information on product life by exposing the product to conditions beyond those of normal design stress. To further streamline this process a two step-stress test will take all elements to failure in a relatively short time. Variables within the sample other than the one that we are controlling in the step-stress testing are uncontrolled but observed and are called covariates. A statistical relationship between the mean lifetime of the test unit and the covariate will allow a prediction of mean lifetime based on the covariate.

In agriculture, animals, or plants may be the test items and dosage of a chemical, amount of fertilizer, temperature, etc may be the stress variable. The breed of the animal or the variety of the plant may be the covariate. In this paper we suggest several potential applications of stepstress testing in agriculture and present inferential procedures for observations that are distributed exponentially.
\end{abstract}




\section{Introduction}

The use of accelerated life testing in industry and agriculture for product testing and development is a time and money saving device to test a product's life in a shorter period of time by shortening that life by exposing the product to a stress beyond its normal design limit. In simple step-stress testing that stress is increased at a predetermined time, $\tau$, with the test run at the second stress level until all test units fail. Step-stress testing not only reduces the time required for testing but also eliminates the need for censoring since the test runs until all the subjects reach failure. Caution must be used in any accelerated life testing that the increased stress does not so drastically change the situation that different systems are affected. For example if we were testing the cool weather tolerance of wheat varieties and our stress levels kept the ground frozen we would not be testing what we set out to test. The efficiency and validity are improved if the time of the stress increase is optimized. Variables within the sample other than the one being controlled in the step-stress testing, such as: manufacturer, day of the week produced, or shelf-life, that are observed but uncontrolled are covariates. A statistical model that relates the covariate and the time to failure in lower or higher stress conditions will allow a life prediction based on the covariate. In this study the effect of the covariate was kept small to simulate the type of differences that would be expected if a product was produced to the same specifications at different plants or by different manufacturers. The interaction of the covariate with the stress increase at $\tau$ has up to this point never been tested for significance or estimated.

\section{Examples in Agriculture}

Example 1. The drought tolerance of two different breeds of soybeans could be tested by growing samples of each under conditions where they received 20\% less water than their normal tolerance. Some plants may stop growing while others survive. The failure time of those plants that stop growing is recorded, and after a predetermined time the water is reduced to $40 \%$ less than normal, testing would end when all the plants stop growing. The covariate would be the two different breeds of soybeans that are tested.

Example 2. CO-OP and Heston brands of baler twine claim to have the same tensile strength. Samples of both are stressed at $200 \%$ of design stress. Some may fail while others survive the failure time is recorded, after a predetermined time the stress is increased to $400 \%$ of design stress and held there until all of the samples fail. The covariate is the brands of twine that are tested.

Example 3. To test the effect of crowding during confinement feeding on two breeds of chickens that have equal size and growth rates, samples of each may be put into conditions where the space per bird is $25 \%$ less than minimal and any birds that die or show less than profitable growth are removed and the time recorded and the space reduced to keep the space per bird constant. After a predetermined time the remaining birds are put into conditions where there space per bird is $50 \%$ less than normal until all the birds die or fail to grow at a profitable rate. The variety of chicken will be the covariate in this test. 


\section{A Mathematical Model for Simple Step-Stress Testing with Covariate}

Procedure for Data Collection

All $n$ test units, $n_{1}$ from covariate group 1 and $n_{2}$ from covariate group 2 where $n_{1}+n_{2}=n$ are initially placed at low stress and run until time $\tau$ when the stress is increased to high stress where the testing is continued until all units fail. We assume that a random number of units $n_{i j}$ from covariate group $j$ fail at stress $x_{i}, i=1,2$ and time to failure $t_{i j k}, k=1,2, \ldots n_{i j}$, are observed and recorded on these test units.

\section{Cumulative Exposure Model}

The model used is termed the cumulative exposure model by Nelson, [1]. The exponential distribution or constant failure rate model will be used to parameterize the data. Let $t$ denote the lifetime of a test unit of interest. The lifetime, $t$ is said to have an exponential distribution if the cumulative distribution function is given by

$$
F(t)=1-\mathrm{e}^{\left(-\frac{t}{\theta}\right)} \text {, where } t>0, \theta>0 .
$$

In applications where the exponential is assumed to model the underlying distribution it is reasonable to assume that the parameter $\theta$ is a function of the stress. The change from design stress in step-stress testing will be reflected in a change in $\theta$ for each new stress level. To analyze data from a step-stress test, one needs a model that relates the life distribution under step-stressing to the distribution under constant stress. The relationship between $\theta$ and stress is given by

$$
\log \left(\theta_{i}\right)=\beta_{0}+\beta_{1} x_{i}
$$

for simple step-stress tests, by

$$
\log \left(\theta_{i j}\right)=\beta_{0}+\beta_{1} x_{i}+\beta_{2} w_{j}
$$

for simple step-stress tests with covariates with no interaction between the covariate and the stress level, and

$$
\log \left(\theta_{i j}\right)=\beta_{0}+\beta_{1} x_{i}+\beta_{2} w_{j}+\beta_{3} x_{i} w_{j}
$$

for simple step-stress tests with covariates with interaction between the covariate and the stress level. In this paper we concentrate on model (3) showing that the interaction between the covariate group and the stress level is statistically significant.

The cumulative exposure model assumes that, at any time, the remaining life of a unit depends only on the exposure it has experienced, and not on how that exposure was accumulated. In other 
words large groups that were brought to the same survivors to originals ratio would have the same life distribution for their surviving members if exposed to the same stress no matter how that ratio was achieved.

For simple step-stress testing the cdf of the time to failure is given by Bia, Kim, Lee, [2]

$$
G(t)=\left\{\begin{array}{ll}
1-e^{-\frac{t}{\theta_{1 j}}} & 0<t<\tau \\
1-e^{-\frac{t-\tau}{\theta_{2 j}}-\frac{\tau}{\theta_{1 j}}} & \tau<t<\infty
\end{array} j=0,1\right. \text { for groups 1 \& 2 respectively }
$$

where $s_{1}$ is the solution of $G_{2}\left(s_{1}\right)=G_{1}(\tau)$.

The basic assumptions of the model are:

(1) Testing is done at stress levels $x_{1}$ and $x_{2}$ where $x_{0} \leq x_{1}<x_{2}, x_{0}$ being the design stress.

(2) The life distribution of a test unit is the exponential model.

(3) The scale parameter $\theta_{i j}$ at stress $x_{i}$ and covariant group $w_{j}$ is given by

$$
\log \left(\theta_{i j}\right)=\beta_{0}+\beta_{1} x_{i}+\beta_{2} w_{j}+\beta_{3} x_{i} w_{j} .
$$

(4) The lifetimes of the test units are independent and identically distributed.

(5) All $\mathrm{n}$ units are initially placed on low stress $x_{1}$ and run until $\tau$ when the stress is increased to $x_{2}$ with testing continuing at $x_{2}$ until all units fail, $\tau$ is the same for both covariate groups.

(6) $\beta_{0}, \beta_{1}, \beta_{2}$, and $\beta_{3}$ are unknown constants: independent of time and stress, and estimated from the test data.

(7) $n_{i 0}+n_{i 1}=n$ where $n_{i 0}, n_{i 1}$ are the number of units in each covariant group and $i$ is the stress level where the unit failed.

Under the assumption of an exponential distribution for $G_{i}$, the likelihood function from observation $t_{i j k}, \mathrm{i}=1,2, k=1,2 \ldots, \mathrm{n}_{\mathrm{ij}}(j=0,1 ; \mathrm{I}=1,0$ for group 1 and group 2 respectively) is:

$$
L\left(\beta_{0,}, \beta_{l}, \beta_{2}, \beta_{3}\right)=\left[\prod_{k=1}^{n 1} \frac{1}{\theta_{l j}} e^{\left(\frac{t_{1 j k}}{\theta_{1 j}}\right)} \prod_{k=1}^{n 2} \frac{1}{\theta_{2 j}} e^{\left(\frac{t_{2 j k}-\tau}{\theta_{2 j}}-\frac{\tau}{\theta_{1 j}}\right)}\right]^{I}\left[\prod_{k=1}^{n 1} \frac{1}{\theta_{l j}} e^{\left(\frac{t_{1 j k}}{\theta_{1 j}}\right)} \prod_{k=1}^{n 2} \frac{1}{\theta_{2 j}} e^{\left(\frac{t_{2, j}-\tau}{\theta_{2 j}}-\frac{\tau}{\theta_{1 j}}\right)}\right]^{(1-I)} .
$$

After simplifications we obtain: 


$$
\begin{aligned}
\log L\left(\beta_{0}, \beta_{1}, \beta_{2}, \beta_{3}\right)= & -n \beta_{0}-\left(n_{10} x_{1}+n_{20} x_{2}+n_{11} x_{1}+n_{21} x_{2}\right) \beta_{1}- \\
& \left(n_{10} w_{0}+n_{20} w_{0}+n_{11} w_{1}+n_{21} w_{1}\right) \beta_{2}- \\
& \left(n_{10} x_{1} w_{0}+n_{20} x_{2} w_{0}+n_{11} x_{1} w_{1}+n_{21} x_{2} w_{1}\right) \beta_{3}+ \\
& I\left[-U_{10} e^{\left(-\beta_{0}-\beta_{1} x_{1}-\beta_{2} w_{0}-\beta_{3} x_{1} w_{0}\right)}-U_{20} e^{\left(-\beta_{0}-\beta_{1} x_{2}-\beta_{2} w_{0}-\beta_{3} x_{2} w_{0}\right)}-\right. \\
& \left.U_{11} e^{\left(-\beta_{0}-\beta_{1} x_{1}-\beta_{2} w_{1}-\beta_{3} x_{1} w_{1}\right)}-U_{21} e^{\left(-\beta_{0}-\beta_{1} x_{2}-\beta_{2} w_{1}-\beta_{3} x_{2} w_{1}\right)}\right]+ \\
& (I-1)\left[-U_{10} e^{\left(-\beta_{0}-\beta_{1} x_{1}-\beta_{2} w_{0}\right)}-U_{20} e^{\left(-\beta_{0}-\beta_{1} x_{2}-\beta_{2} w_{0}\right)}-\right. \\
& \left.U_{11} e^{\left(-\beta_{0}-\beta_{1} x_{1}-\beta_{2} w_{1}\right)}-U_{21} e^{\left(-\beta_{0}-\beta_{1} x_{2}-\beta_{2} w_{1}\right)}\right]
\end{aligned}
$$

where $n=n_{1, j}+n_{2, j}$ and

$$
\log \left(\theta_{i j}\right)=\beta_{o}+\beta_{l} x_{i}+\beta_{2} w_{j}+\beta_{3} x_{i} w_{j}
$$

where

$$
U_{10}=\sum_{k=1}^{n_{10}} t_{k 0}+n_{20} \tau \quad U_{20}=\sum_{k=1}^{n_{20}}\left(t_{k 0}-\tau\right) \quad U_{11}=\sum_{k=1}^{n_{11}} t_{k 1}+n_{21} \tau \quad U_{21}=\sum_{k=1}^{n_{21}}\left(t_{k 1}-\tau\right) .
$$

To find the maximum likelihood estimates, $\hat{\beta}_{0}, \hat{\beta}_{1}, \hat{\beta}_{2}$, and $\hat{\beta}_{3}$, for the model parameters $\beta_{0}, \beta_{1}, \beta_{2}$, and $\beta_{3}$ we need to solve the following system of equations:

$$
\begin{aligned}
& \frac{\partial \log L\left(\beta_{0}, \beta_{1}, \beta_{2}, \beta_{3}\right)}{\partial \beta_{0}}=- n+I\left[U_{10} e^{\left(-\beta_{0}-\beta_{1} x_{01}-\beta_{2} w_{0}-\beta_{3} x_{1} w_{0}\right)}+U_{20} e^{\left(-\beta_{0}-\beta_{1} x_{02}-\beta_{2} w_{0}-\beta_{3} x_{2} w_{0}\right)}+\right. \\
&\left.U_{11} e^{\left(-\beta_{0}-\beta_{1} x_{01}-\beta_{2} w_{1}-\beta_{3} x_{1} w_{1}\right)}+U_{21} e^{\left(-\beta_{0}-\beta_{1} x_{12}-\beta_{2} w_{1}-\beta_{3} x_{2} w_{1}\right)}\right]+ \\
&(I-1)\left[U_{10} e^{\left(-\beta_{0}-\beta_{1} x_{01}-\beta_{2} w_{0}-\beta_{3} x_{1} w_{0}\right)}+U_{20} e^{\left(-\beta_{0}-\beta_{1} x_{02}-\beta_{2} w_{0}-\beta_{3} x_{2} w_{0}\right)}+\right. \\
&\left.U_{11} e^{\left(-\beta_{0}-\beta_{1} x_{01}-\beta_{2} w_{1}-\beta_{3} x_{1} w_{1}\right)}+U_{21} e^{\left(-\beta_{0}-\beta_{1} x_{12}-\beta_{2} w_{1}-\beta_{3} x_{2} w_{1}\right)}\right]=0 \\
& \frac{\partial \log L\left(\beta_{0}, \beta_{1}, \beta_{2}, \beta_{3}\right)}{\partial \beta_{1}}=-\left(n_{10} x_{1}+n_{20} x_{2}+n_{11} x_{1}+n_{21} x_{2}\right)+I\left[x_{1} U_{10} e^{\left(-\beta_{0}-\beta_{1} x_{01}-\beta_{2} w_{0}-\beta_{3} x_{1} w_{0}\right)}+\right. \\
& x_{2} U_{20} e^{\left(-\beta_{0}-\beta_{1} x_{02}-\beta_{2} w_{0}-\beta_{3} x_{2} w_{0}\right)}+x_{1} U_{11} e^{\left(-\beta_{0}-\beta_{1} x_{11}-\beta_{2} w_{1}-\beta_{3} x_{1} w_{1}\right)}+ \\
&\left.x_{2} U_{21} e^{\left(-\beta_{0}-\beta_{1} x_{12}-\beta_{2} w_{1}-\beta_{3} x_{2} w_{1}\right)}\right]+(I-1)\left[x_{1} U_{10} e^{\left(-\beta_{0}-\beta_{1} x_{01}-\beta_{2} w_{0}-\beta_{3} x_{1} w_{0}\right)}+\right. \\
& x_{2} U_{20} e^{\left(-\beta_{0}-\beta_{1} x_{02}-\beta_{2} w_{0}-\beta_{3} x_{2} w_{0}\right)}+x_{1} U_{11} e^{\left(-\beta_{0}-\beta_{1} x_{11}-\beta_{2} w_{1}-\beta_{3} x_{1} w_{1}\right)}+ \\
&\left.x_{2} U_{21} e^{\left(-\beta_{0}-\beta_{1} x_{12}-\beta_{2} w_{1}-\beta_{3} x_{2} w_{1}\right)}\right]=0
\end{aligned}
$$




$$
\begin{aligned}
\frac{\partial \log L\left(\beta_{0}, \beta_{1}, \beta_{2}, \beta_{3}\right)}{\partial \beta_{2}}= & -\left(n_{10} w_{0}+n_{10} w_{0}+n_{11} w_{1}+n_{21} w_{1}\right)+I\left[w_{0} U_{10} e^{\left(-\beta_{0}-\beta_{1} x_{01}-\beta_{2} w_{0}-\beta_{3} x_{1} w_{0}\right)}+\right. \\
& w_{0} U_{20} e^{\left(-\beta_{0}-\beta_{1} x_{02}-\beta_{2} w_{0}-\beta_{3} x_{1} w_{0}\right)}+w_{1} U_{11} e^{\left(-\beta_{0}-\beta_{1} x_{11}-\beta_{2} w_{1}-\beta_{3} x_{1} w_{1}\right)}+ \\
& \left.w_{1} U_{21} e^{\left(-\beta_{0}-\beta_{1} x_{12}-\beta_{2} w_{1}-\beta_{3} x_{2} w_{1}\right)}\right]+(I-1)\left[w_{0} U_{10} e^{\left(-\beta_{0}-\beta_{1} x_{01}-\beta_{2} w_{0}-\beta_{3} x_{1} w_{0}\right)}+\right. \\
& w_{0} U_{20} e^{\left(-\beta_{0}-\beta_{1} x_{02}-\beta_{2} w_{0}-\beta_{3} x_{1} w_{0}\right)}+w_{1} U_{11} e^{\left(-\beta_{0}-\beta_{1} x_{11}-\beta_{2} w_{1}-\beta_{3} x_{1} w_{1}\right)}+ \\
& \left.w_{1} U_{21} e^{\left(-\beta_{0}-\beta_{1} x_{12}-\beta_{2} w_{1}-\beta_{3} x_{2} w_{1}\right)}\right]=0
\end{aligned}
$$

$\frac{\partial \log L\left(\beta_{0}, \beta_{1}, \beta_{2}, \beta_{3}\right)}{\partial \beta_{3}}=-\left(n_{10} x_{1} w_{0}+n_{20} x_{2} w_{0}+n_{11} x_{1} w_{1}+n_{21} x_{2} w_{1}\right)+$

$$
\begin{gathered}
I\left[x_{1} w_{0} U_{10} e^{\left(-\beta_{0}-\beta_{1} x_{01}-\beta_{2} w_{0}-\beta_{3} x_{1} w_{0}\right)}+x_{2} w_{0} U_{20} e^{\left(-\beta_{0}-\beta_{1} x_{02}-\beta_{2} w_{0}-\beta_{3} x_{1} w_{0}\right)}+\right. \\
\left.x_{1} w_{1} U_{11} e^{\left(-\beta_{0}-\beta_{1} x_{11}-\beta_{2} w_{1}-\beta_{3} x_{1} w_{1}\right)}+x_{2} w_{1} U_{21} e^{\left(-\beta_{0}-\beta_{1} x_{12}-\beta_{2} w_{1}-\beta_{3} x_{2} w_{1}\right)}\right]+ \\
(I-1)\left[x_{1} w_{0} U_{10} e^{\left(-\beta_{0}-\beta_{1} x_{01}-\beta_{2} w_{0}-\beta_{3} x_{1} w_{0}\right)}+x_{2} w_{0} U_{20} e^{\left(-\beta_{0}-\beta_{1} x_{02}-\beta_{2} w_{0}-\beta_{3} x_{1} w_{0}\right)}+\right. \\
\left.x_{1} w_{1} U_{11} e^{\left(-\beta_{0}-\beta_{1} x_{11}-\beta_{2} w_{1}-\beta_{3} x_{1} w_{1}\right)}+x_{2} w_{1} U_{21} e^{\left(-\beta_{0}-\beta_{1} x_{12}-\beta_{2} w_{1}-\beta_{3} x_{2} w_{1}\right)}\right]=0
\end{gathered}
$$

The Fisher information matrix is obtained by taking the negative expected values of the second partial and mixed partial derivatives of $\log L\left(\beta_{0}, \beta_{1}, \beta_{2}, \beta_{3}\right)$ with respect to $\beta_{0}, \beta_{1}, \beta_{2}$, and $\beta_{3}$. The Fisher Information matrix is used to determine the optimal value of $\tau$. Running the test with optimal $\tau$ minimizes the asymptotic variance of the log mean lifetime at the design stress. The Fisher information matrix is:

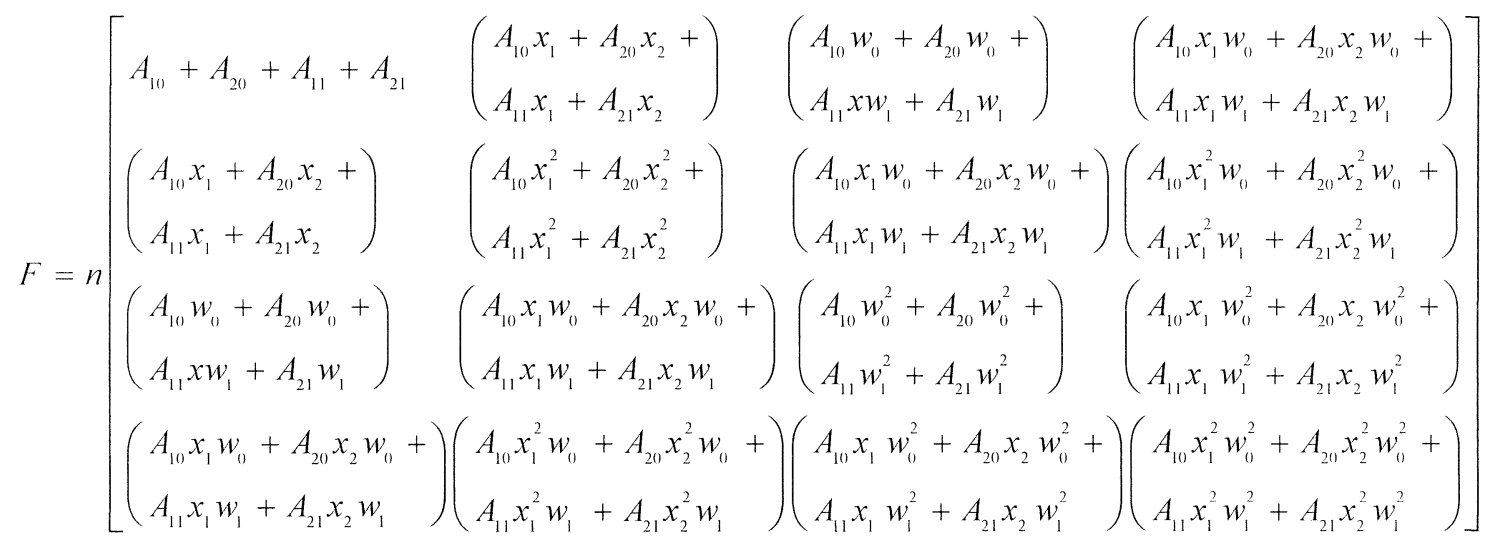

Where $\left(A_{10}+A_{11}\right)=1-e^{\left(-\frac{\tau}{\theta_{1 j}}\right)} \quad A_{10}, A_{11}$ are the probabilities that a test unit fails while at stress $x_{1}$ for each covariate group $\left(A_{20}+A_{21}\right)=1-\left(A_{10}+A_{11}\right) A_{20}, A_{21}$ are the probabilities that a test unit fails at stress $x_{2}$ for each group, and $A_{10}+A_{20}+A_{11}+A_{21}=1$ 


\section{A Numerical Example}

Suppose a researcher wishes to test the two brands of twine as described earlier in Example 2. The stress could be standardized so that the design stress for the twine, $100 \%$, is represented by $x_{0}=0$, the low stress level, $200 \%$, by $x_{1}=1$, and the high stress, $400 \%$, by $x_{1}=2$. The stress values will be model parameters that would have to be determined by an engineer based on how the baler twine reacts to increased stress. The $\tau$ is a time when we would expect 50 percent of the samples to have failed at low stress. The quantitative variables, $w_{0}=0$ and $w_{1}=1$ are used to distinguish between the brands of twine within the formula. If we were running an actual test we might take 20 samples of each brand, 20 pieces of CO-OP twine and 20 pieces of Heston twine, and put all 40 samples under a stress of $200 \%$ of their maximum design stress. We would note the time that each sample failed until we reach the predetermined time to change the stress to $400 \%$ of design stress noting the failure times until all samples failed. Because this type of research has not yet been done in agriculture, for this paper, we created a computer generated simulation, a brief description of that simulation follows.

A simulation of a simple step-stress test with covariate and interaction was run with a sample size of forty units, twenty from each covariate group. The following values were assumed for the simulation. The same value for $\tau$ is used for each covariate group.

$$
\begin{aligned}
& x_{0}=0, x_{1}=1, x_{2}=2 \\
& \beta_{0}=0, \beta_{1}=1, \beta_{2}=0.01, \beta_{3}=0.02 \\
& w_{0}=0, w_{1}=1 \\
& \tau=2.50
\end{aligned}
$$

To run the simulation two groups of twenty random numbers between zero and one were generated to represent the probabilities that a test unit failed at time $t$. The equation:

$$
\begin{gathered}
t= \begin{cases}-\theta_{1, j} \ln [1-G(t)] & 0<t<\tau \\
\tau-\frac{\theta_{2, j}}{\theta_{1 j}} \tau-\theta_{2, j} \ln [1-G(t)] & \tau \leq t \leq \infty\end{cases} \\
\log \left(\theta_{i j}\right)=x_{i}+0.01 w_{j}+0.02 x_{i} w_{j}
\end{gathered}
$$

was used to generate the simulated failure times listed in table one. The values from this

simulation allow the solving for $\hat{\beta}_{0}, \hat{\beta}_{1}, \hat{\beta}_{2}$, and $\hat{\beta}_{3}$, the maximum likelihood estimates based on the data. The maximum likelihood estimates of the model parameters are:

$\hat{\beta}_{0}=0.474, \hat{\beta}_{1}=0.711, \hat{\beta}_{2}=-0.080, \hat{\beta}_{3}=-0.156$. 
The optimum time to change the stress from the lower accelerated level to the higher one is the time that minimizes the asymptotic variance of the log mean failure time at the design stress. The asymptotic variance multiplied by the sample size $n$ is given by the equation:

$$
n A V=n(1,0,1,0) F^{-1}(1,0,1,0)^{T}
$$

Mathematica was used to find the value of $\tau$ that minimizes $n A V$ to determine the optimal time for $G$; that optimal $\tau$ was found to be 3.08. Figure 1 gives evidence that the value $\tau=3.08$ is a unique solution for the minimum value of $n A V$.

\section{Statistical Analysis of the Results}

Tests of hypotheses about parameters of the model can be obtained by using the likelihood ratio method. An important inference problem concerning the regression coefficients $\left(\beta_{0}, \beta_{1}, \beta_{2}, \beta_{3}\right)$ is the test of hypothesis $H_{0}: \beta_{3}=0$ against $H_{1}: \beta_{3} \neq 0$. To test $H_{0}$ against $H_{1}$ one can use the likelihood ratio statistic Khamis, [3]

$$
\Lambda=-2 \log \left[\frac{L\left(\beta_{0}, \beta_{1}, \beta_{2}, 0\right)}{L\left(\hat{\beta_{0}}, \hat{\beta_{1}}, \hat{\beta_{2}}, \hat{\beta}_{3}\right)}\right]
$$

where $\beta_{0}, \beta_{1}, \beta_{2}$ are the MLEs of $\beta_{0}, \beta_{1}, \beta_{2}$ under $H_{0}, \beta_{3}=0$, and $\hat{\beta}_{0}, \hat{\beta}_{1}, \hat{\beta}_{2}, \hat{\beta}_{3}$, are the unrestricted MLEs. Large values of $\Lambda$ provide evidence against $H_{0}$, and approximate significance levels can be calculated by using the fact that in large samples $\Lambda$ is approximately distributed as $\chi^{2}$ under $H_{0}$. Using the data from the previous simulations a value for $\Lambda$ can be obtained. The data from Table 1 and the log likelihood equation were used along with the MLEs.

$$
\begin{gathered}
\Lambda=-2\left[\log L\left(\beta_{0}, \beta_{1}, \beta_{2}, 0\right)-\log L\left(\hat{\beta_{0}}, \hat{\beta_{1}}, \hat{\beta_{2}}, \hat{\beta}_{3}\right)\right] \\
\Lambda=-2[-102.299-(-95.345)] \\
\Lambda=13.908
\end{gathered}
$$

With one degree of freedom $\chi_{(0.05)}^{2} \approx 3.841, \alpha=0.05$ significance level, the value $\Lambda=13.908$ is significantly larger than the $\chi^{2}{ }_{(0.05)}$ with one degree of freedom value so we reject the hypothesis $H_{0}: \beta_{3}=0$. This indicates that the interaction of the covariate with the stress level is significant.

\section{Conclusion.}

Accelerated life testing has long been accepted as a reliable method for testing product life but the interaction of a covariate with stress level is a previously untested idea. In this study the differences between the covariate subgroups was kept small as was the interaction between the covariate subgroups and the stress levels. This was done to reflect the use of this model in industrial or agricultural testing where the covariate subgroups could be circuit boards made to the same specifications by different suppliers or grasses of the same variety but one containing 
an engineered genetic mutation, which would allow one variety to be unaffected by a specific herbicide. The exponential model with covariates with interaction between the covariate subgroups and the stress level can be used as a predictor of the life of the elements of the subgroups. Further work in this area might include extension to more than two covariate groups, and using distribution models other than the exponential.

\begin{tabular}{ll} 
& \multicolumn{1}{c}{ Notation } \\
$x_{0}, x_{1}, x_{2}$ & design, low, and high stress levels \\
$w_{j}$ & covariate group; $j=0$ and $w_{j}=0$ for covariate group $1, j=1$ and $w_{j}=1$ \\
& for covariate group 2 \\
$x_{i} w_{j}$ & the interaction between stress level and covariate group \\
$\theta_{i j}$ & scale parameter for stress $x_{i}$ in covariate group $w_{j}$ \\
$n$ & total number of units in the test group \\
$n_{i j}$ & number of units that failed at stress level $i$ in covariate group $j, n_{i}$ if no \\
$t_{i j}$ & covariate \\
$\tau$ & time of unit failure at stress level $i$ and covariate group $j, t_{i}$ if no covariate \\
$\tau^{*}$ & time of stress change from $x_{1}$ to $x_{2}$ \\
$\beta$ & optimal time to change stress from $x_{1}$ to $x_{2}$ \\
$\hat{\beta}$ & model parameters \\
$A V$ & maximum likelihood estimates of model parameters \\
cdf & asymptotic variance of the log mean time at design stress \\
$G(t)$ & cumulative distribution function \\
MLE & cdf of a test unit under simple step-stress test \\
$I$ & maximum likelihood estimate \\
& group indicator variable $I=1$ for group 1 and $I=0$ for group 2
\end{tabular}

Table 1 Simulated failure time data for each covariate group

\begin{tabular}{|c|c|c|c|c|c|c|}
\hline Group 1 & Failure Time & & Group 2 & Failure Times & & \\
\hline $\begin{array}{l}\text { Low } \\
\text { stress } \\
x_{1}=1\end{array}$ & $\begin{array}{ll}0.085 & 0.271 \\
1.095 & 1.346 \\
1.800 & 2.734\end{array}$ & $\begin{array}{ll}0.283 & 0.571 \\
1.437 & 1.670 \\
2.443 & \end{array}$ & $\begin{array}{l}\text { Low } \\
\text { stress } \\
x_{1}=1\end{array}$ & $\begin{array}{ll}0.058 & 0.058 \\
0.517 & 0.946 \\
1.516 & 1.845\end{array}$ & $\begin{array}{l}0.330 \\
1.212 \\
1.953\end{array}$ & $\begin{array}{l}0.511 \\
1.430 \\
2.472\end{array}$ \\
\hline
\end{tabular}




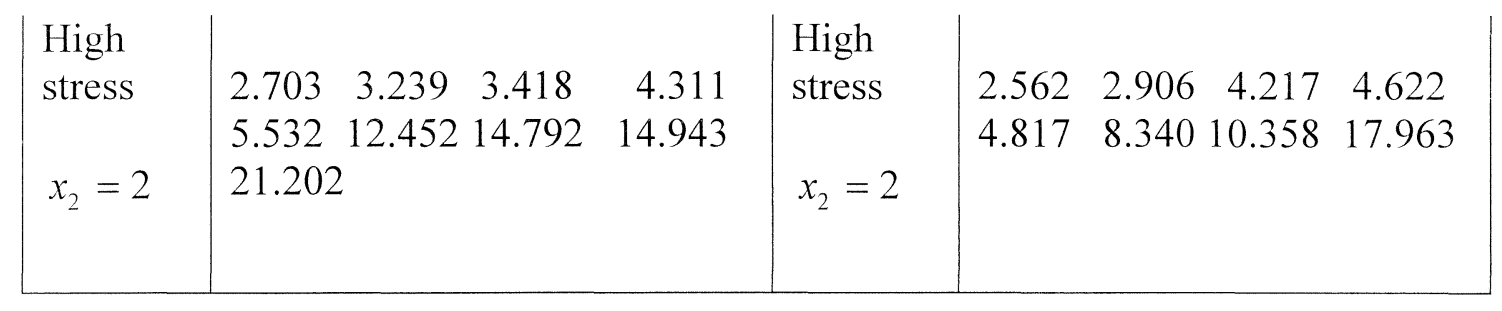

Figure 1 Asymptotic variance as a function of $\tau$

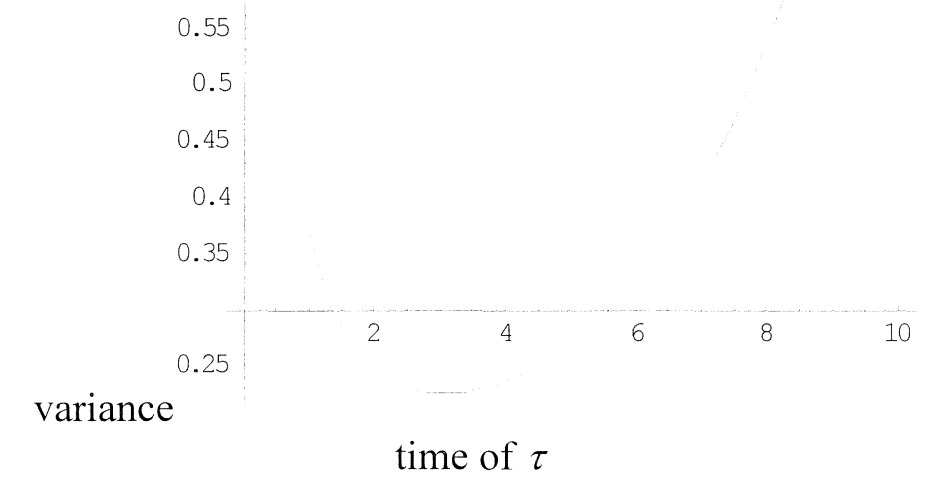

\section{References}

[1] W. Nelson, 'Accelerated Testing Statistical Models, Test Plans, and Data Analysis', Wiley, New York, 1990.

[2] D.S. Bia, M.S. Kim, S.H. Lee, 'Optimum simple step-stress accelerated life test with censoring,' IEEE Transactions on Reliability, Vol. R-38, December, 1989.

[3] I.H. Khamis, 'Multiple Step-Stress Testing,' unpublished PhD Thesis, Kansas State University, 1996. 